\title{
Velocity-less imaging of linearly inhomogeneous media in 3D
}

Andrej Bóna ${ }^{1 *}$, Dennis Cooke ${ }^{2}$

CurtinUniversity,a.bona@curtin.edu.au ${ }^{1}$,Santos,dennis.cooke@santos.com ${ }^{2}$

Key Words: imaging, velocity-less migration, slowness and traveltime inversion.

\section{SUMMARY}

We show that in a medium with constant velocity-gradient it is possible to find both the two velocity parameters, and the locations of the reflector for each source-receiver pair from the reflection traveltimes. This algorithm is a basis for a velocity-less migration; it allows for using the obtained velocity parameters and reflection position for migrating the data. The presented method relies on obtaining the derivatives of the traveltimes with respect to the locations of the sources and receivers in the two horizontal directions.

\section{INTRODUCTION}

It is possible to find the velocity and the reflector in below a constant velocity layer by using the traveltimes and the horizontal slownesses of along the reflected rays. Ottolini (1983) first presented the idea of using local slopes for velocity-less migration. More recently, Fomel (2007) has extended these concepts to perform hyperbolic NMO, DMO and velocity-less PSTM. Cooke et al. (2008) present another point of view on this velocity-less migration using curvelets.

Herein, we generalise this approach to layers with constant velocity-gradients. To do so, we need to consider enough equations for the unknowns: coordinates of the reflector, velocity at zero depth and the velocity gradient. This number of equations is different for two-dimensional and three-dimensional surveys. In the case of a two-dimensional survey, one needs four equations to find the two coordinates of the reflector and the two velocity parameters. In the case of a three-dimensional survey, one needs five equations for the three reflector coordinates and two for the velocity parameters. The important distinction between the two and three-dimensional cases is the availability of the equations. In the two-dimensional case we can use the reflected traveltimes, the direct-arrival traveltime and one horizontal slowness at the source and another at the receiver. In the three-dimensional case we can use the reflected traveltime and the two components of the horizontal slowness at both the source and the receiver. In this presentation we will focus on the three-dimensional case.

\section{THEORY}

Herein, we consider signal propagation in one layer with a constant velocity-gradient: layer in which the signal velocity increases linearly with depth, namely $v(z)=a+b z$. The shape of raypaths in such media is circular, with the centre of the sections of the circles at height $a / b$ above the plane $z=0$. We start our discussion with the analytical expressions for the traveltime.

Following e.g. Červený (2001), we can express the traveltime of a reflected signal from location $(x, y, z)$ as

$$
\begin{aligned}
t= & \frac{1}{b} \operatorname{arccosh}\left(1+b^{2} \frac{\left(x-x_{s}\right)^{2}+\left(y-y_{s}\right)^{2}+z^{2}}{2 a(a+b z)}\right) \\
& +\frac{1}{b} \operatorname{arccosh}\left(1+b^{2} \frac{\left(x-x_{r}\right)^{2}+\left(y-y_{r}\right)^{2}+z^{2}}{2 a(a+b z)}\right),
\end{aligned}
$$

where $\left(x_{s}, y_{s}\right)$ are the source coordinates and $\left(x_{r}, y_{r}\right)$ are the receiver coordinates. Using expression (1), we can express the two horizontal components of the slowness at the source and receiver as

$$
p_{s x}:=\frac{\partial t}{\partial x_{s}}=\frac{-2\left(x-x_{s}\right)}{\sqrt{R_{s}^{2}+z^{2}} \sqrt{2 c+b^{2}\left(R_{s}^{2}+z^{2}\right)}}
$$

$$
\begin{aligned}
& p_{s y}:=\frac{\partial t}{\partial y_{s}}=\frac{-2\left(y-y_{s}\right)}{\sqrt{R_{s}^{2}+z^{2}} \sqrt{2 c+b^{2}\left(R_{s}^{2}+z^{2}\right)}} \\
& p_{r x}:=\frac{\partial t}{\partial x_{r}}=\frac{-2\left(x-x_{r}\right)}{\sqrt{R_{r}^{2}+z^{2}} \sqrt{2 c+b^{2}\left(R_{r}^{2}+z^{2}\right)}}
\end{aligned}
$$

and

$$
p_{r y}:=\frac{\partial t}{\partial y_{r}}=\frac{-2\left(y-y_{r}\right)}{\sqrt{R_{r}^{2}+z^{2}} \sqrt{2 c+b^{2}\left(R_{r}^{2}+z^{2}\right)}},
$$

where we replaced velocity $a$ with

$$
c:=2 a(a+b z),
$$

and used notation

$$
R_{S}:=\sqrt{\left(x-x_{S}\right)^{2}+\left(y-y_{S}\right)^{2}}
$$

and

$$
R_{r}:=\sqrt{\left(x-x_{r}\right)^{2}+\left(y-y_{r}\right)^{2}} .
$$

The horizontal slownesses can be obtained from the measured traveltime. Multiplying equation (1) by $b$ and applying hyperbolic cosine, we obtain

$$
\begin{aligned}
& \cosh (b t)=\left(1+\frac{b^{2}\left(R_{s}^{2}+z^{2}\right)}{c}\right)\left(1+\frac{b^{2}\left(R_{r}^{2}+z^{2}\right)}{c}\right)+ \\
& +\frac{b^{2} \sqrt{\left(R_{s}^{2}+z^{2}\right)\left(R_{r}^{2}+z^{2}\right)\left(2 c+b^{2}\left(R_{s}^{2}+z^{2}\right)\right)\left(2 c+b^{2}\left(R_{r}^{2}+z^{2}\right)\right)}}{c^{2}} .
\end{aligned}
$$

Combining equation (2) with (3) and equation (4) with (5), we write

$$
\frac{-2\left(y-y_{s}\right)}{p_{s y}}=\sqrt{R_{s}^{2}+z^{2}} \sqrt{2 c+b^{2}\left(R_{s}^{2}+z^{2}\right)}=\frac{-2\left(x-x_{s}\right)}{p_{s x}}
$$


and

$$
\frac{-2\left(y-y_{r}\right)}{p_{r y}}=\sqrt{R_{r}^{2}+z^{2}} \sqrt{2 c+b^{2}\left(R_{r}^{2}+z^{2}\right)}=\frac{-2\left(x-x_{r}\right)}{p_{r x}},
$$

from which we find coordinates $x$ and $y$ of the reflector:

$$
x=\frac{p_{s x} p_{r x}\left(y_{r}-y_{s}\right)-p_{s x} p_{r y} x_{r}+p_{r x} p_{s y} x_{s}}{p_{s y} p_{r x}-p_{r y} p_{s x}}
$$

and

$$
y=\frac{p_{r y} p_{s y}\left(x_{r}-x_{s}\right)-p_{r x} p_{s y} y_{r}+p_{r y} p_{s x} y_{s}}{p_{r y} p_{s x}-p_{s y} p_{r x}} .
$$

This leaves us with finding the three remaining unknowns; $z, b$ and $c$. Adding squares of equations (2) and (3), we write for the horizontal slowness $P_{S}$ at the source

$P_{s}^{2}:=p_{s x}^{2}+p_{s y}^{2}=\frac{4\left(r_{s}^{2}-z^{2}\right)}{r_{s}^{2}\left(2 c+b^{2} r_{s}^{2}\right)}=\frac{4 R_{s}^{2}}{\left(R_{s}^{2}+z^{2}\right)\left(2 c+b^{2}\left(R_{s}^{2}+z^{2}\right)\right)}$.

Similarly, we write the square of the receiver horizontal slowness as

$$
P_{r}^{2}:=p_{r x}^{2}+p_{r y}^{2}=\frac{4\left(r_{r}^{2}-z^{2}\right)}{r_{r}^{2}\left(2 c+b^{2} r_{r}^{2}\right)}=\frac{4 R_{r}^{2}}{\left(R_{r}^{2}+z^{2}\right)\left(2 c+b^{2}\left(R_{r}^{2}+z^{2}\right)\right)} .
$$

From these equations we write

$$
\begin{aligned}
& 2 c=\frac{4 R_{s}^{2}}{\left(R_{s}^{2}+z^{2}\right) P_{s}^{2}}-b^{2}\left(R_{s}^{2}+z^{2}\right) \\
& 2 c=\frac{4 R_{r}^{2}}{\left(R_{r}^{2}+z^{2}\right) P_{r}^{2}}-b^{2}\left(R_{r}^{2}+z^{2}\right) .
\end{aligned}
$$

Equating the right-hand sides of these equations yields expression for $b^{2}$

$$
b^{2}=4 \frac{R_{r}^{2}\left(R_{s}^{2}+z^{2}\right) P_{s}^{2}-R_{s}^{2}\left(R_{r}^{2}+z^{2}\right) P_{r}^{2}}{\left(R_{r}^{2}-R_{s}^{2}\right)\left(R_{r}^{2}+z^{2}\right)\left(R_{s}^{2}+z^{2}\right) P_{s}^{2} P_{r}^{2}} .
$$

After substituting expression (11) in the sum of equations (9) and (10), we obtain

$$
c=2 \frac{R_{s}^{2}\left(R_{r}^{2}+z^{2}\right)^{2} P_{r}^{2}-R_{r}^{2}\left(R_{s}^{2}+z^{2}\right)^{2} P_{s}^{2}}{\left(R_{r}^{2}-R_{s}^{2}\right)\left(R_{s}^{2}+z^{2}\right)\left(R_{r}^{2}+z^{2}\right) P_{r}^{2} P_{s}^{2}} .
$$

We can solve the nonlinear traveltime equation (6) for $z$ by substituting expressions (11) and (12) for $b$ and $c$ by using numerical methods. This way, we find the last coordinate of the reflector. This allows us to find for each reflector $b$ and $c$ by substituting the reflector coordinates to expressions (11) and (12). These two numbers give us the velocity field $v(z)=a+b z$, by solving for $a$ from $c$.

There are several methods for finding the horizontal slownesses and the second derivatives of the traveltimes. These methods can be split into two categories: instantaneous and spectral. Spectral methods allow for multiple values of the slowness. The instantaneous methods can be:

- Any of the numerical differentiation methods applied to picked traveltime.

- Plane-wave destruction as discussed by Fomel (2002).
- Quotient of instantaneous spatial and temporal frequencies, as discussed by Tanner et al. (1979).

Some of the spectral methods are:

- A collection of fan filters applied in the FK domain, as discussed by Cooke et al. (2008).

- Curvelet Transform as per Douma and de Hoop (2007).

- Any time shift method calculating coherency or similarity between a window on adjacent traces for different time shifts between those windows.

Since a part of the purpose of this work is to show applicability of the presented new method in various circumstances, for the example in the following section we choose the slowness finding method that is in many ways the least robust - numerical differentiation of the picked traveltimes.

\section{EXAMPLE}

We demonstrate the above described imaging and velocity determination method on the following three-dimensional velocity model. We consider a medium with a constant horizontal velocity gradient $v(z)=(1500+0.8 z) m s^{-1}$ and irregular reflector, as illustrated on Figure 1 .

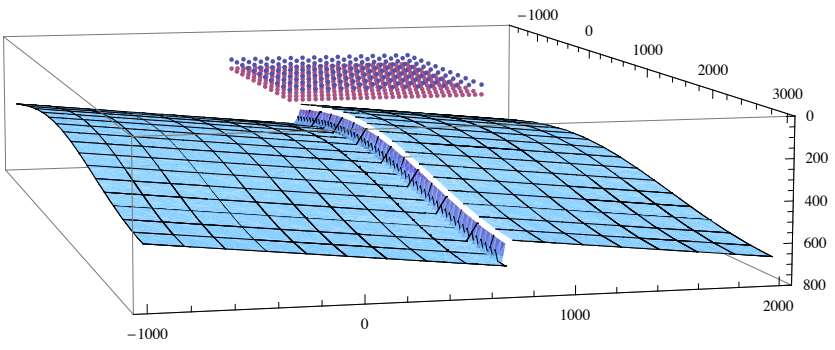

Figure 1: Constant velocity gradient layer with velocity $(1500+0.8 z) \mathrm{ms}^{-1}$ and nonlinear interface. Locations of the sources are indicated by blue dots and of the receivers by purple dots. The sources and receivers are displayed with vertical separation for clarity.

The reflected traveltimes are represented by the three-dimensional graphs on Figure 2, where the horizontal axes correspond to the location of the source and receiver. We used only the first arrivals, but we could have used multiple slownesses to find the corresponding multiple reflection locations for a given source-receiver pair. An example of the computed slownesses is shown on Figure 3, where the horizontal axes correspond to the location of the receiver. We note that $p_{s}\left(x_{s}, y_{s}, x_{r}, y_{r}\right)=p_{r}\left(x_{r}, y_{r}, x_{s}, y_{s}\right)$ due to the symmetry of the traveltime $t\left(x_{s}, y_{s}, x_{r}, y_{r}\right)=t\left(x_{r}, y_{r}, x_{s}, y_{s}\right)$. From the computed reflection points we eliminated the outliers; the results are 


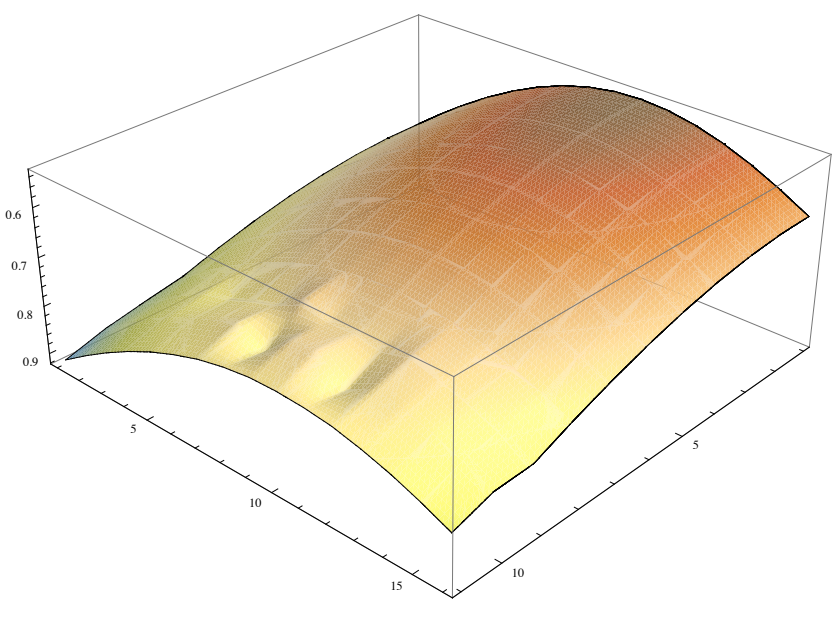

Figure 2: The reflected traveltimes in seconds from a fixed source as a function of the receiver positions. The numbers on the horizontal axes correspond to the receiver numbers.

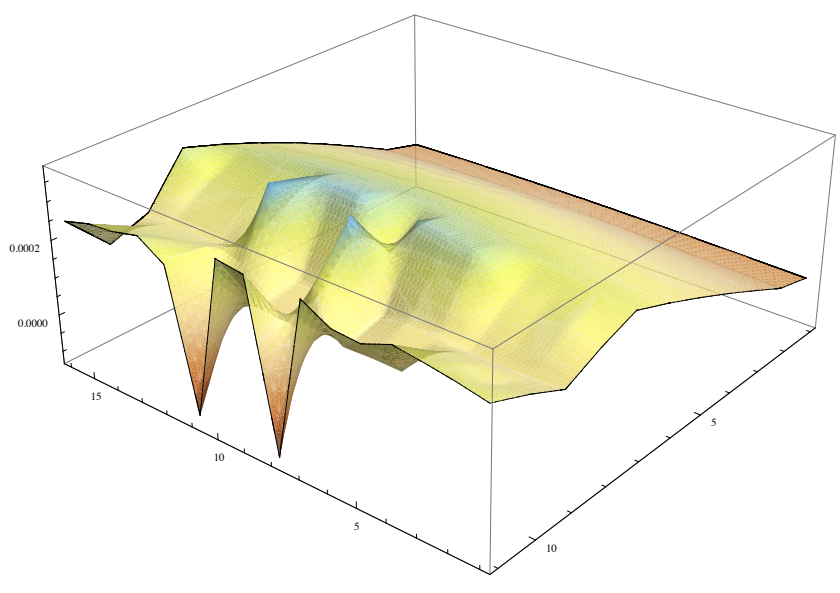

Figure 3: The horizontal slowness $p_{s x}$ as a function of the receiver position.

presented on Figure 4. The computed reflection points that are not matching the true reflector can be traced to the source-receiver pairs for which one of the four slowness components exhibits nonsmoothness, as exemplified by Figure 3. This nonsmoothness can be in turn traced to the errors in the traveltime picking shown on Figure 2.

The values of the velocity parameters can be averaged for the entire layer, or assigned to each source-receiver pair. Such a distribution of the computed velocities can be useful for slowly laterally changing velocities, as discussed in Conclusions. The median values of the velocity parameters computed from all source-receiver pairs are $\bar{a}=1343 m s^{-1}$ and $\bar{b}=0.9 \mathrm{~s}^{-1}$. Since we considered laterally homogeneous layer, in this particular case there is no advantage in assigning the computed values of the velocity parameters to the corresponding reflection locations or source-receiver pairs.

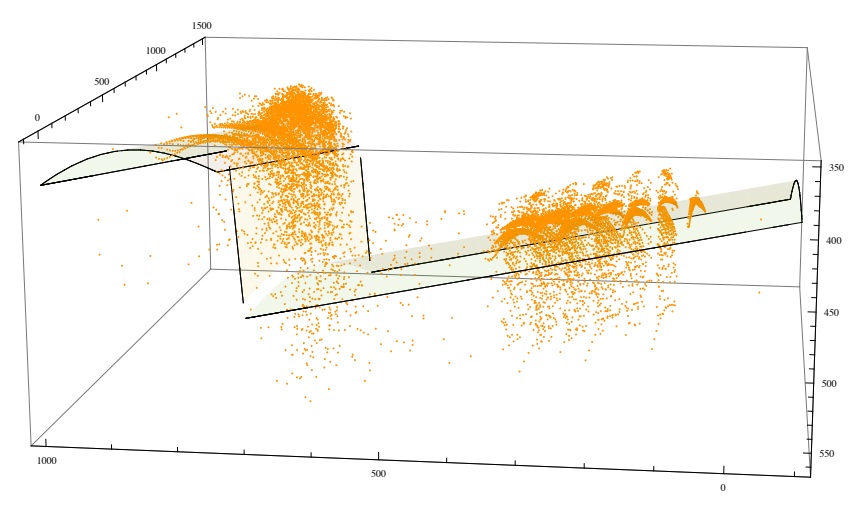

Figure 4: The computed reflection points overlayed on the true reflector.

\section{CONCLUSIONS}

We describe a method of directly finding the velocity of a laterally homogeneous medium with a constant velocity gradient together with the reflection position for each source-receiver pair. This method makes use of the horizontal components of the slowness at the source and receiver locations. In a three-dimensional case, these components together with the reflected traveltime provide us with enough data to find the velocity parameters and the reflector position. However, in a two-dimensional case, there are only two horizontal components of the slownesses. Thus, we need to provide another set of data. This can be either the direct traveltime or a second derivative of the reflected traveltime with respect to position of the source or receiver.

The presented example shows the sensitivity of the inversion to the estimation of the slownesses. Figure 3 demonstrates how a small error in traveltime picking, shown in Figure 2, influences horizontal slownesses. This sensitivity suggests that smoothing of the slownesses should be applied to improve the results of the inversion.

The presented method yields not only location of the reflector, but also provides the velocity and its gradient. This is beneficial for imaging in layered media, where the constant velocity gradient model fits better the reality than the standard constant RMS velocity model. A generalization of Dix's approach using our method is a part of the future work focused on imaging of more complex media. Applications of the presented method for laterally varying media should be also possible, due to the fact that we can compute the velocity properties for each source-receiver pair. 


\section{REFERENCES}

Červený, V., 2001, Seismic ray theory: Cambridge University Press.

Cooke, D., A. Bóna, and B. Hansen, 2008, Simultaneous time imaging, velocity estimation and multiple suppression using local event slopes: Presented at the Annual SEG conference.

Douma, H. and M. de Hoop, 2007, Leading-order seismic imaging using curvlets.: Geophysics, 72, S231-S248.

Fomel, S., 2002, Applications of plane-wave destruction filters.: Geophysics, 67, 1946-1960.

,2007, Velocity-independent time-domain seismic imaging using local event slopes: Geophysics, 72, S139-S147.

Ottolini, R., 1983, Velocity independent seismic imaging: Stanford Exploration Project, 37, 59-68.

Tanner, M., F. Kochler, and R. Sherrif, 1979, Complex seismic trace analysis.: Geophysics, 44, 1041-1063. 